\title{
Computer-Aided Education for Magnetostatics
}

\author{
François Buret, Member, IEEE, Daniel Muller, and Laurent Nicolas
}

\begin{abstract}
We present in this paper a computer-aided education (CAE) package allowing to visualize the magnetostatic field in and around the magnetic circuit of a double- $U$ shaped contactor. The modeling of this device is performed by solving in real time a nonlinear finite-element problem. The implementation of this method is optimized greatly in order to allow to animate field patterns in real time in reaction to any user interaction. An evaluation of the improvements brought by this technique to the learning capabilities of students is presented.
\end{abstract}

Index Terms - Contactors, education, finite-element methods, magnetostatics.

\section{INTRODUCTION}

W E HAVE already presented the COLOS project in previous papers [1], [2]. It is composed of research teams of ten universities located in seven different countries. Its major goal is to encourage the development of teaching methods so as to improve the knowledge and the understanding of basic concepts. Special focus is made on intuitive and qualitative approaches, more particularly in science and technology.

The package Contact described here has been developed at CEGELY within this project. It is used by students in engineering at Ecole Centrale de Lyon, in conjunction with an introductory course to electrical engineering. The objective of Contact is to allow the students to intuitively understand how the magnetic field arranges inside a magnetic circuit when varying parameters such as the inductor current, the number of turns of the inductor coil, the airgap width, and even the material characteristics. Contact takes into account the nonlinearity of the materials, and enables therefore to study the influence of the saturation of the magnetic circuit.

The main problem when designing this package was to give an instant feedback to any interaction with the student: in other words, any modification of a geometric, electric, or magnetic parameter has to induce in real time a modification of the displayed results. The mathematical model used to get the distribution of the magnetic field is computed with the finite-element method (FEM) for two main reasons. First, the local magnetic field has to be represented in the whole studied domain. Second, the characteristic of the magnetic materials may be nonlinear. Great optimization of its implementation enabled then a real-time solving. This is the key point of our approach: great accuracy of the displayed results is not necessary. On the other hand, fast solving time is required in order to allow a real feedback.

Manuscript received October 25, 1995; revised October 29, 1998.

The authors are with CEGELY, UPRESA CNRS 5005, Ecole Centrale de Lyon BP163, 69131 Ecully cedex, France.

Publisher Item Identifier S 0018-9359(99)01238-8.

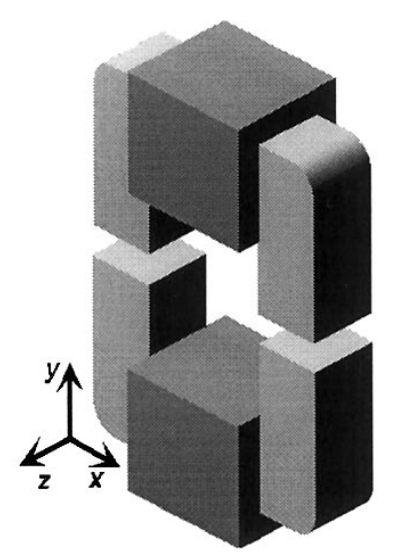

Fig. 1. Geometry of the magnetic circuit of the contactor.

We describe in a first part the studied device, the results that the students visualize, and what they are supposed to understand. In a second part, we present the FEM applied to two-dimensional (2-D) magnetostatics, and how we were able to compute the distribution of the magnetic field in real time. We describe then the practical work that the students have to perform. Finally, we show how the pedagogical efficiency of the package has been analyzed.

\section{GRAPHIC INTERFACE AND OBJECTIVES OF THE APPLICATION}

The studied device is the magnetic circuit of a double- $U$ shaped contactor (Fig. 1). The magnetic field is created by one or two dc coils located on each arm of the contactor.

Because of the large depth of the device, 2-D approximation can be made (Fig. 2): the magnetic field is assumed to be located in the vertical $x y$-cross section, and the edge effects in the $z$-direction perpendicular to the cross section are neglected.

Using the corresponding arrow buttons (Fig. 3), the user may modify geometrical parameter (the airgap), electrical parameters (the current and the number of turns of both coils), and magnetic parameters (the relative permeability at the origin of the B-H curve, and the flux density at saturation for both arms of the contactor).

As results (Fig. 4), the students visualize the amplitude of the flux density, represented with color shading. They may also superimpose field lines. Contact gives them also access to a real-time updated table of results, showing the field value at a few characteristic points, the flux through the airgap surface, and other meaningful quantities such as energy or force.

With this type of application, we are looking for the students to: 


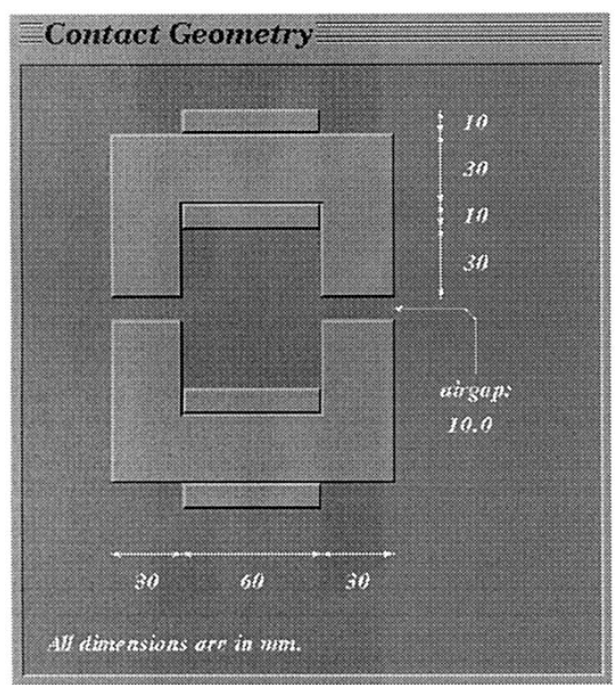

Fig. 2. Studied geometry.

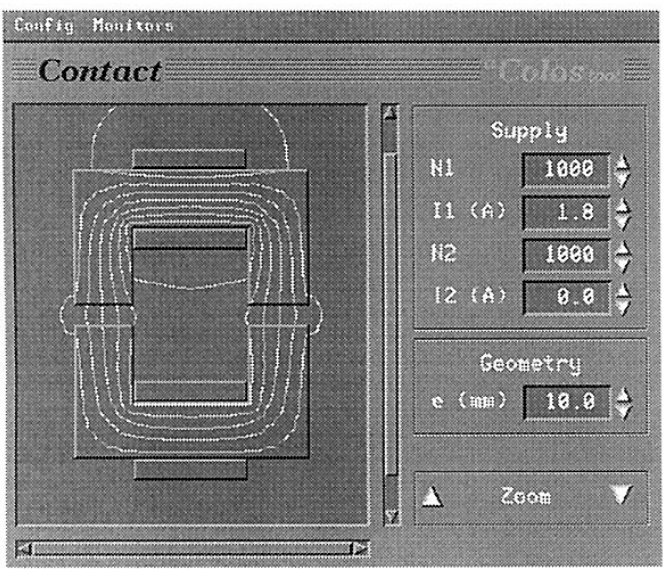

Fig. 3. Contact user interface: main window.

- understand the magnetostatic phenomena, including the influence of the saturation;

- get the range values of these phenomena;

- understand some physical concepts, such as the Ampere law.

\section{The Finite-Element Model}

The main goal of computer-aided education is to offer the students a highly interactive environment allowing them to examine any desired configuration of the studied device with instant feedback. Taking into account the nonlinearity of the materials made unavoidable the use of the FEM.

\section{A. The Finite-Element Method for Magnetostatics}

From the Maxwell equations, a magnetostatics problem is expressed in term of the vector magnetic potential $\mathbf{A}$

$$
\nabla \times \frac{1}{\mu} \nabla \times \mathbf{A}=\mathbf{J}
$$

where $\mu$ is the magnetic permeability and $\mathbf{J}$ is the source current density.
In 2-D, the vector potential $\mathbf{A}$ has only one component $A_{z}$ perpendicular to the studied cross section. This leads to the following nonlinear equation:

$$
-\frac{\partial}{\partial x}\left(\frac{1}{\mu} \frac{\partial A_{z}}{\partial x}\right)-\frac{\partial}{\partial y}\left(\frac{1}{\mu} \frac{\partial A_{z}}{\partial y}\right)=J_{z} .
$$

Using energy-based arguments, it can be shown that solving (2) may be performed by minimizing the energy functional (3) (in the next expression, $\mu$ is assumed to be constant per region). This is called the variational approach

$$
\mathrm{L}\left(A_{z}\right)=\iint\left\{\frac{1}{2 \mu}\left[\nabla A_{z}\right]^{2}-J_{z} A_{z}\right\} d R .
$$

The variational approach to finite elements [3] consists then in dividing the solution region into elements (FE), in defining interpolating functions for the unknown values of $A_{z}$, in putting these functions into the energy functional (3), in deriving this expression in order to minimize it, and solving the so-obtained linear system.

The nonlinear equation is linearized by using the chord method [3]. A relaxation factor is introduced to force the convergence. This method is generally seen as highly inefficient, since the convergence depends on how close the initial guess is to the actual solution. However the variations of the parameters are always smooth due to the interactivity with the user. Therefore the actual solution is never far away from the initial guess, and the number of iterations is then reduced.

\section{B. Optimization of the Solver}

The FEM by itself is well known for such an analysis and does not lead to any problem. We worked mainly at the implementation of the FEM, which has been optimized as much as possible in order to minimize the solving times and to get a real-time feedback. One can mention:

- the discretization is performed using first-order triangles;

- the problem is described with one symmetry;

- the structure of the mesh does not vary: only the vertical coordinates of the nodes may change, because of the possible modification of the airgap;

- the structure of the FE matrix is then prestored;

- all the local submatrices are precomputed, except the ones corresponding to the airgap;

- the nested dissection renumbering algorithm [4] is used to solve the system matrix;

- in nonlinear cases, the solution corresponding to the previous state of the magnetic circuit is taken as initial solution, decreasing then the number of iterations.

The mesh (Fig. 5) is made of 468 triangles and 270 nodes. On a mid-range workstation with a 75-Mips computation power (HP9000/712), a linear solution takes about $30 \mathrm{~ms}$ to be computed, and a nonlinear solving goes up to $250 \mathrm{~ms}$ : this is compatible with the maximum delay required by a smooth animation. 


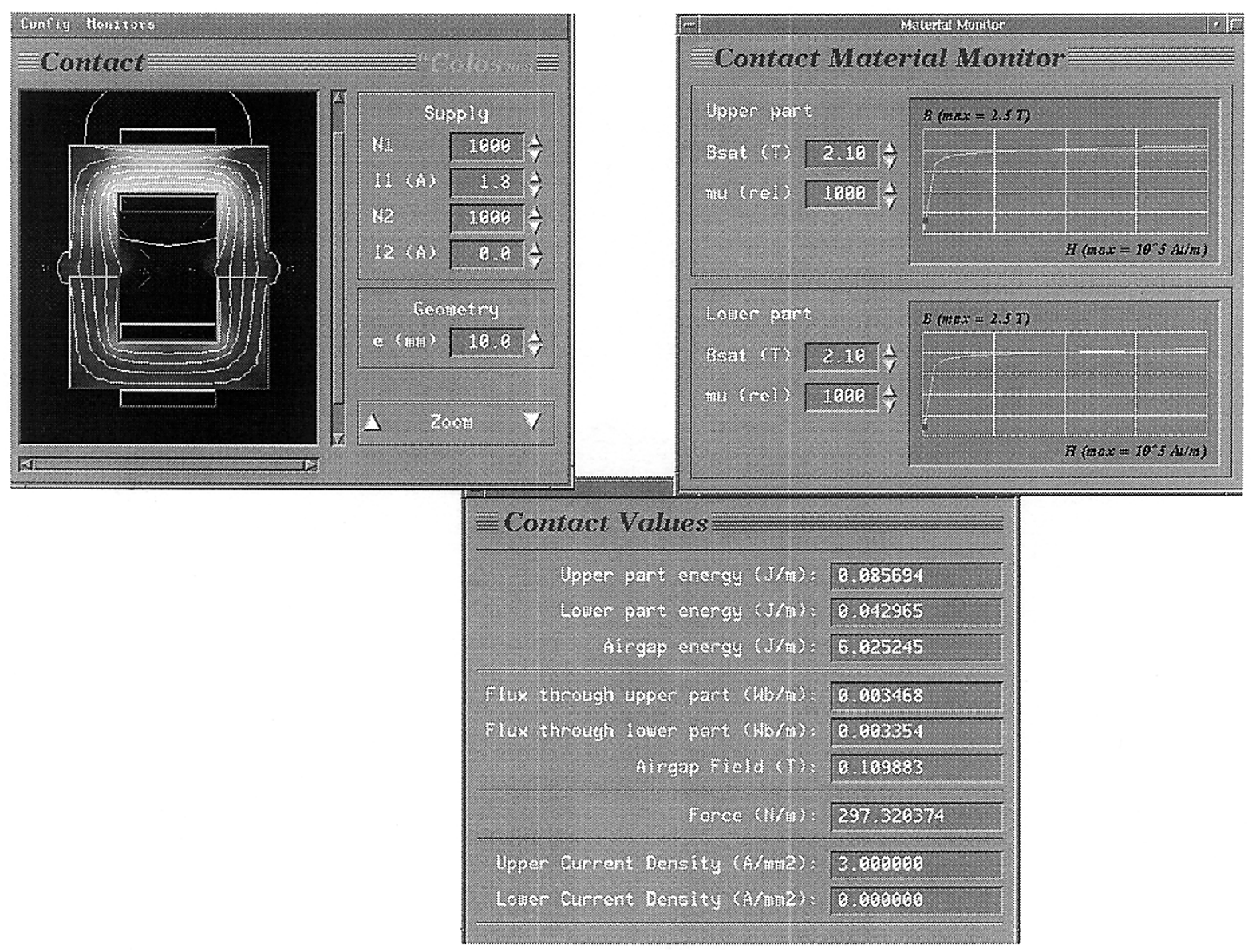

Fig. 4. Contact complete user interface: main window, window for the monitoring of the magnetic materials, and table of results.

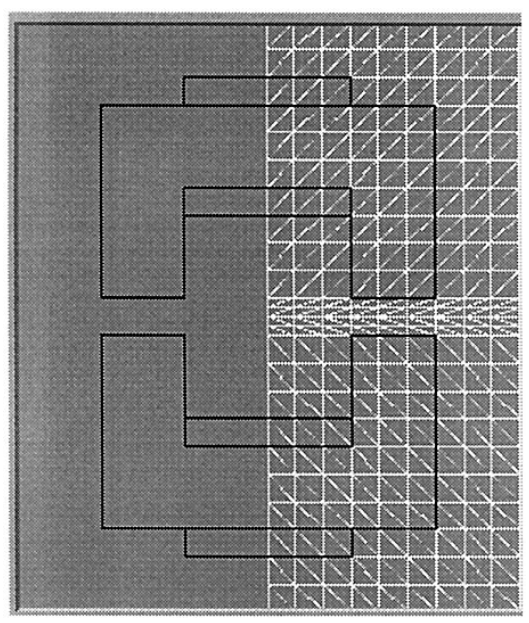

Fig. 5. Mesh used for the FE computation.

\section{Guidelines for the Practical Work}

A guide is provided to the students to perform the practical work. First, this guide proposes them to examine qualitatively several aspects of electromagnetism.

- The influence of the permeability on the distribution and on the value of induction lines when currents are constant, magnetic materials non saturated and without airgap.
- The influence of the width of the airgap on the induction value at a given current. This influence is studied for small and large values of magnetic material permeability.

- The evolution of the magnetic state of the circuit when the sum of the ampere-turns remains constant, but distributed in different manners (remind that the number of turns and intensity of both coils can vary independently).

- The influence of the saturation of one arm of the circuit on the flux. The parameter settings are chosen in order to strongly saturate one material, the other parameters can vary.

Quantitative aspects are also approached in a second step. For different values of the airgap and permeabilities, students have to evaluate:

- the distribution of magnetic energy between the air (airgap) and the magnetic materials;

- the relationship between the flux in the magnetic core and the current (Fig. 6);

- the variation of the force in relation with the flux.

\section{EVALUATION}

In order to test the pedagogical efficiency of this new approach, we have made an evaluation of the students through a multiple-choice questionnaire (MCQ). Note that the notions used during the practical work have been previously exposed 


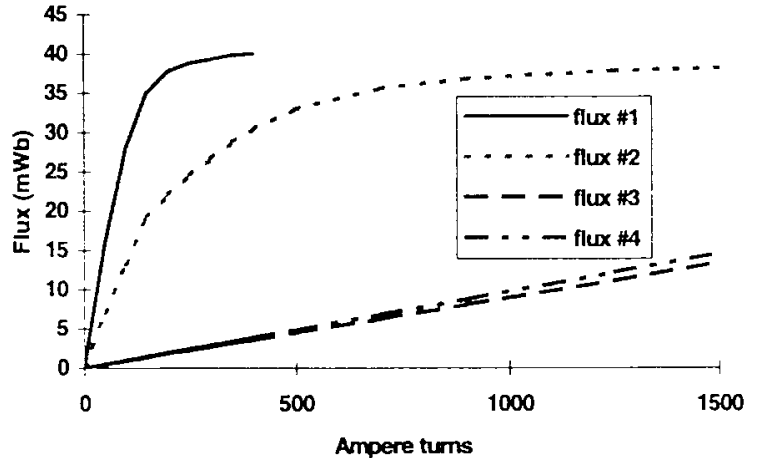

Fig. 6. Example of relation deduced by the students. Flux versus current, for different values of airgap and permeability. 1: airgap $=0.1 \mathrm{~mm}, \mu r=10000$ 2: airgap $=0.1 \mathrm{~mm}, \mu r=1000 ; 3:$ airgap $=4 \mathrm{~mm}, \mu r=1000 ;$ and 4 : airgap $=4 \mathrm{~mm}$.

during a classical lesson. Therefore students should be able to reply correctly to all questions.

\section{A. The Multiple-Choice Questionnaire}

We have asked students to reply to a MCQ at the beginning and at the end of the session. Questions carry on theoretical aspects of magnetostatics and on aspects linked to orders of magnitudes that appear in the practice. This MCQ is composed by the ten next affirmations:

1) in a magnetic circuit with a large airgap, the magnetic energy is essentially located in the iron;

2) the Ampere's law is only true for linear materials;

3) a high permeability magnetic circuit canalizes the lines of the B-field;

4) with given designing data, the magnetic field only depends on the sum of the ampere-turns;

5) with a large airgap the flux does not dependent on the value of the relative permeability (if it is higher than 1000);

6) the relation flux versus current is nonlinear if materials present a saturation of the $\mathrm{B}(\mathrm{H})$ curve;

7) a large airgap makes difficult the saturation of a magnetic circuit;

8) the attraction force between two parts of a magnetic circuit is proportional to $B^{2}$;

9) when one of the arms of the magnetic circuit is saturated, the flux does not vary mainly in function of the other parameters (current, permeability, ....);

10) with no airgap and small current, the induction field is proportional to the relative permeability.

The students have to tell if each of these affirmations are true, false or if they ignore the answer. The scores are established by counting +1 for a correct answer, -1 for a false answer, or zero if no answer. Hence the score can range between -10 and +10 .

\section{B. Analysis of the Results}

Answers concern a population of 221 students, which is significant on a statistical point of view. These results can first be analyzed globally. Fig. 7 shows the distribution of students

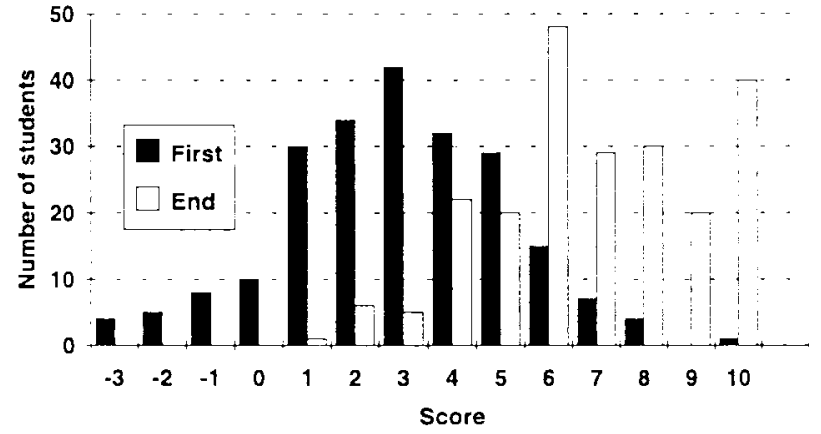

Fig. 7. Evolution of the score of the students between the beginning and the end of the practical work.

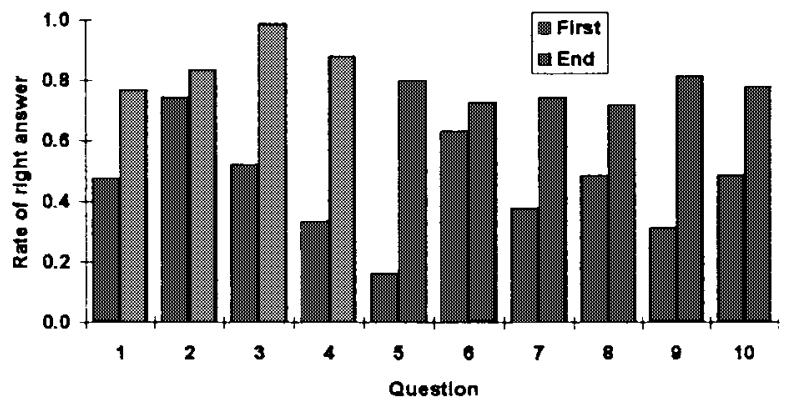

Fig. 8. Rate of good answers for each question.

versus the score that they have obtained at the beginning and at the end of the session. One hundred ninety-nine students progress while 11 have stable results and 11 regress. At the end of the session there is a 4-point increase of the mean value of the scores. Indeed this mean value rises from 2.9 to 6.9. The population scoring five or more increases from 56 to 187 individuals. Forty students obtain the maximal score at the end while only one gets it at the beginning. Undoubtedly the work performed by the students using this package improves strongly their level of knowledge.

The evolution of scores may also be analyzed on the basis of each question. It allows to test the pertinence of the questions. Fig. 8 shows the rate of good answers (number of good answers divided by the total number of answers):

- At the beginning of the session the average rate is about $45 \%$ with a large dispersion depending on the questions: for example, question 2 has a success rate close to $75 \%$ while question 5 does not exceed $16 \%$.

- At the end of the session, about $80 \%$ of students answer correctly. This rate fluctuates only a little with the questions: from $72 \%$ for question 8 to $99 \%$ for the question 3 .

Fig. 9 shows the efficiency of the work session. It is calculated as the number of students which switch from a false answer to a correct answer, divided by the number of false answers at the beginning of the work. Questions 2 and 6 show a low efficiency because they have a good initial success rate. This is not surprising: the notions approached in these questions are simply deduced from classical laws of electromagnetism, and students are supposed to know them since a long time. On the other hand, question 5 shows a 


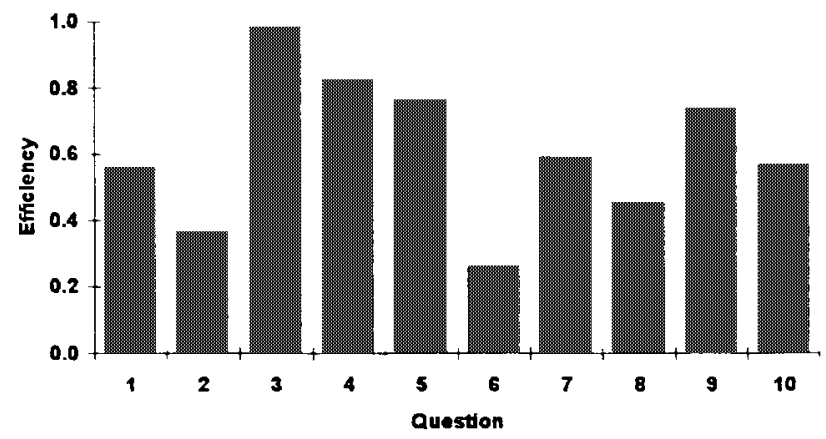

Fig. 9. Efficiency of the work session.

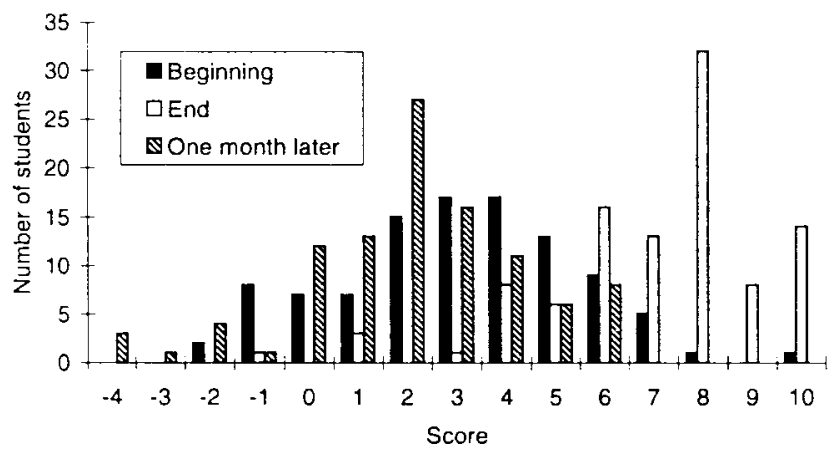

Fig. 10. Evolution of the score of the students between the beginning and the end of the practical work and one month later.

good efficiency, which is also not surprising: it deals with a quantitative aspect of electromagnetism, which is less known by students.

Obviously the Contact package allows the students to understand quickly the electromagnetic phenomena occurring in an magnetic device. There is however no guarantee that the learning is definitively digested. As example, Fig. 10 shows the results obtained with another group of students (102 students) the next year. This figure has to be compared to Fig. 7: it represents the score obtained at the beginning and at the end of the practical session, and one month later. This group obtains results comparable with the previous one, since this mean value rises from 3.1 to 7.1 (instead of 2.9 to 6.9). However, one month later, the mean value decreases to 2.1, which is below the original value!

\section{CONCLUSION}

We have described in this paper a CAE package for nonlinear magnetostatics. This package is based on the FEM which has been greatly optimized in order to obtain fast computation times. From a pedagogical point of view, it seems to be really efficient when used in parallel with a classical lecture. It is, however, obvious that this package does not exempt the student to have a regular and durable work.

\section{REFERENCES}

[1] H. Härtel, "The project COLOS-Conceptual Learning of Science-Objectives, background, and first results," COLOS Project Internal Paper, Kiel, Jan. 1993.

[2] D. Muller, L. Nicolas, and A. Nicolas, "A C.A.E. package for an intuitive approach to microwaves," IEEE Trans. Magn., vol. 30, pp. 3216-3219, Sept. 1994.

[3] S. R. H. Hoole, Computer-Aided Analysis and Design of Electromagnetic Devices. New York: Elsevier, 1989.

[4] A. George and J. W. Liu, Computer Solution of Large Sparce Positive Definite Systems. Englewood Cliffs, NJ: Prentice-Hall, 1981.

François Buret (M'95) was born in Avignon, France, on August 10, 1955. He received the bachelor's degree from the "Ecole Normale Supérieure de l'Enseignement Technique" (ENS Cachan) and the Ph.D. degree from the Institut National Polytechnique de Grenoble, France, in 1986.

His interests include specially high-voltage insulation and power apparatus. Since 1982, he has been teaching and researching in the Electrical Engineering Department of "Ecole Centrale de Lyon" (CNRS UPRESA 5005), France.

Daniel Muller is Professeur Agrege in electrical engineering and teaches electrical engineering since 1984 at Ecole Centrale de Lyon, France, where he is also Information and Communication Technology Advisor since 1998. First pursuing his research at CEGELY then at ICTT (Interactions Collaboratives, Teleactivites, Teletravail), he is now involved with the application of information and communication technology to teaching. He is also working for the COLOS (COnceptual Learning Of Science) project, part of which are ten European universities and the Hewlett-Packard Company, where he is in charge of computer-aided teaching tools development.

Laurent Nicolas received the bachelor's degree from the Ecole Normale Supérieure d'Ingénieurs Electriciens de Grenoble, France.

He is currently a Researcher at the CNRS, in the Centre de Génie Electrique de Lyon (CNRS UPRESA 5005, Ecole Centrale de Lyon), France. His interests include modeling of electromagnetic phenomena using numerical methods (finite-element method, boundary integral equation method). 